\title{
Radio Resource Management in a Coordinated Cellular Distributed Antenna System By Using Particle Swarm Optimization
}

\author{
Omer Haliloglu ${ }^{(1)}$, Cenk Toker ${ }^{(1)}$, Gurhan Bulu ${ }^{(1)}$, Halim Yanikomeroglu ${ }^{(2)}$ \\ ${ }^{(1)}$ Department of Electrical and Electronics Engineering, Hacettepe University, Ankara, Turkey \\ ${ }^{(2)}$ Department of Systems and Computer Engineering, Carleton University, Ottawa, Canada \\ Email: \{omer, cenk.toker, bulu\}@ee.hacettepe.edu.tr, halim@sce.carleton.ca
}

\begin{abstract}
In this paper, we consider a coordinated multi-point transmission (CoMP) scheme used in a cellular system where antenna ports are distributed throughout the cell, instead of using a single base station. Two schemes are considered; either ports can be switched on and off (Binary Power Management, BPM) or their transmission power can be adjusted (Continuous Power Management, CPM). The goal is to maximize the minimum signal to interference plus noise ratio (SINR) in the network for both schemes.

The first problem is NP-hard and the second one is multimodal. We propose to use particle swarm optimization (PSO) as a solver for both problems. It is demonstrated that the proposed PSO based algorithms can efficiently solve both problems. Furthermore, through simulations, it is shown that for the same total transmit power per port, CPM outperforms BPM.
\end{abstract}

Index Terms - Distributed antenna systems, CoMP, port selection, radio resource management, particle swarm optimization.

\section{INTRODUCTION}

The ever increasing user demand from wireless communication systems in terms of both data rate and also coverage has been the underlying motivation of the search for advanced technologies. Traditional interference avoidance techniques which reuse frequency and time resource blocks to mitigate interference, utilize spectrum inefficiently and can be inadequate to meet the demand for higher data rates. SINR degradation due to signal attenuation and interference from other cells for cell edge users can be considered as a coverage issue. Furthermore, using transmit power efficiently is important due to both system performance and environmental aspects.

It appears that all these issues cannot be simultaneously addressed and solved by the techniques which have been used in the last decades. Future developments will tend to be based on cooperative transmission rather than interference avoidance, leading to coordinated multi-point transmission and reception (CoMP) [1]. CoMP is a promising technique for communication networks, especially in heterogeneous networks (HetNets) [2], due to the deployment of low power nodes, i.e., pico-, femto-cells and remote radio heads. CoMP offers self-optimization and self-configuration functionality for

This work is supported by TUBITAK (The Scientific and Technological Research Council of Turkey), Turkey, under project no. 112E024. network operators and the direction of the future evolution of LTE-A seems to be guided by CoMP.

In this paper, a downlink CoMP scenario is considered, and ports (remote radio heads in LTE-A terminology) are utilized to form a distributed antenna system. It is assumed that inter-user interference in a cell is eliminated by assigning the resource blocks (RBs) to the users of the cell in an orthogonal fashion, i.e., in each cell only one user equipment (UE) is allowed to use a specific RB. On the other hand, the transmission of the ports using the same RB in different cells can interfere each other. To simplify the formulation and demonstration, transmission over a single RB is considered in this paper.

Authors in [3] investigate a similar scenario. The objective of the problem in [3] is to maximize the minimum SINR among all users by decreasing the best port settings. They handle this problem by setting the ports either on or off, i.e., a port can either transmit at a fixed power or it is switched off. We call this scheme as Binary Power Management (BPM). The problem is NP-hard [5] and need to be relaxed before it can be solved by an optimization tool, e.g., CVX [7]. Semidefinite relaxation (SDR) is a good way to tackle NP-hard problems [6], but it gives suboptimum results and can become not promising for more complex problems.

In this paper, we allow ports to transmit at a power level in the interval $\left[0, P_{\max }\right]$ rather than $\left\{0, P_{\max }\right\}$ as in [3]. We call this scheme as Continuous Power Management (CPM). It can be shown that although the search space is continuous, the cost function is multi-modal, i.e., there can be many local optimum solutions besides the global one. Therefore it is important to find the global optimum solution giving the best setup.

In order to obtain the optimum solution, we offer to use particle swarm optimization (PSO) which is an evolutionary optimization method first proposed in [8]. The most important feature of PSO is, it distributes the particles (i.e., possible solutions) throughout the search space and performs a global search. As in many other evolutionary optimization algorithms, PSO also does not suffer from getting stuck at a local optimum, if it is set up correctly.

If the peak power constraint for a port is considered, it is demonstrated that the solution to both the BPM and CPM 


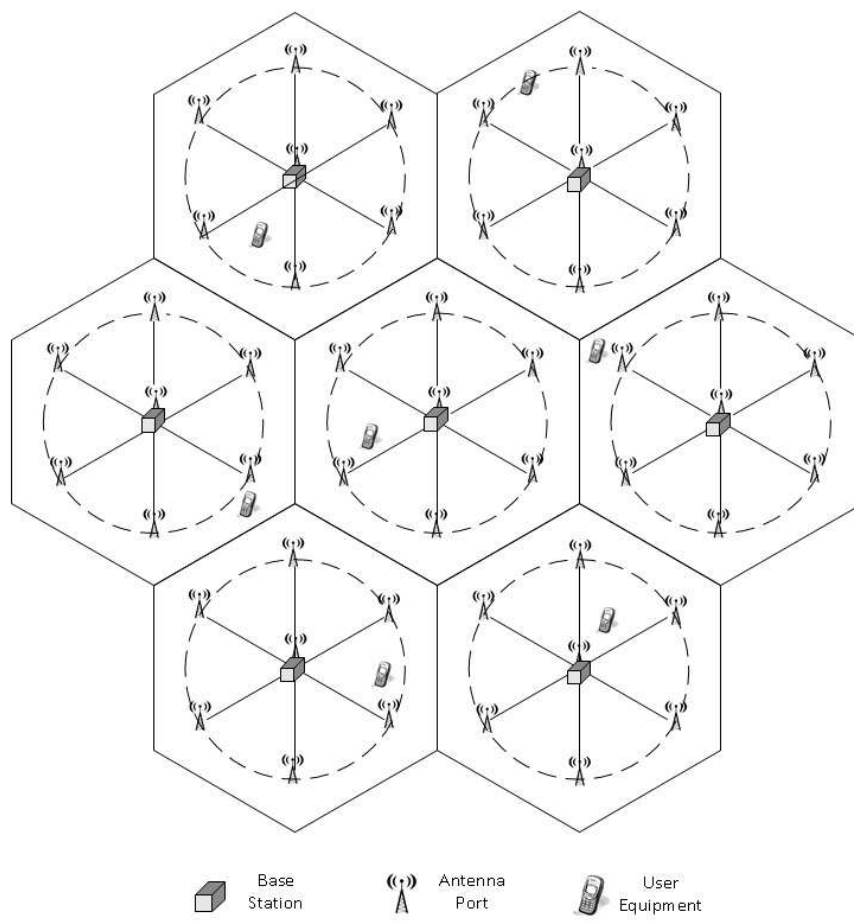

Fig. 1: A seven cell distributed antenna system with seven ports per cell.

problems can be classified into two parts; a noise dominant region, and an interference-limited region. For a small network of two cells, these regions are apparent. However as the network size, i.e., number of cells in the network increases, the solution gets interference-limited for physically meaningful scenarios. Through simulations it is shown that CPM outperforms BPM for both a small and also a relatively large network, especially in the interference-limited region.

The paper is organized as follows; Section II describes the system model. Particle swarm optimization is introduced in Section III. Complexity analysis and simulation results are examined in Section IV and Section V. Section VI concludes the paper.

\section{System Model}

Consider the scenario depicted in Figure 1, where there are $M$ cells, and each cell contains $L$ distributed single-antenna ports connected to each other via high speed communication links, giving $M \times L$ ports in total. All ports in a cell transmit the same signal simultaneously. It is assumed that the transmit power of each port can be adjusted independently.

The same RB can be used throughout the network, however at most one user is allowed to access the same RB within a cell. A user can communicate with the ports in its dedicated cell, whereas the signals from other cells are considered as interference. Resource management throughout the network is conducted by a central network entity.
Let $x_{m}$ be the information signal for the user in the $m$ th cell, where $E\left\{x_{m} x_{n}\right\}=\delta_{m n}$ and $\delta_{m n}=1$ when $m=$ $n$, and zero otherwise. The complex-valued coefficients $h_{l n m}$ represent the channel gain between the $l$-th port of the $n$-th cell and the UE in the $m$-th cell for $l=1, \ldots, L$ and $m=$ $1, \ldots, M$. The zero mean circularly symmetric additive white Gaussian noise with variance $\sigma_{n}^{2}$ for the UE in the $m$-th cell is denoted by $n_{m}$. Each port has its own peak power value given by $P_{l m}$ (which can be taken as $P_{\max }$ network-wide for simplicity) and the transmit power for the $l$-th port in the $m$-th cell is controlled by the power coefficients $\alpha_{l m} \in\{0,1\}$ for BPM, and $\alpha_{l m} \in[0,1]$ for CPM. Furthermore, let $w_{l m}$ be the complex beamsteering coefficient for the $l$-th port in the $m$-th cell. Then the received signal of the UE in the $m$-th cell can be written as

$$
\begin{aligned}
y_{m}= & \sum_{l=1}^{L} \alpha_{l m} \sqrt{P_{l m}} h_{l m m} w_{l m} x_{m}+ \\
& \sum_{n=1, n \neq m}^{M} \sum_{l=1}^{L} \alpha_{l n} \sqrt{P_{l n}} h_{l n m} w_{l n} x_{n}+n_{m}, \quad \forall_{m} .
\end{aligned}
$$

The expression in the first line provides the signal intended to the user in the $m$-th cell whereas the second line contains the interference from the other cells and also noise.

Eventually, the corresponding SINR for the UE in the $m$-th cell can be expressed as

$$
\begin{aligned}
& \operatorname{SINR}_{m}(\boldsymbol{\alpha}, \mathbf{w})= \\
& \frac{\left|\sum_{l=1}^{L} \alpha_{l m} \sqrt{P_{l m}} h_{l m m} w_{l m}\right|^{2}}{\sigma_{n}^{2}+\sum_{n=1, n \neq m}^{M}\left|\sum_{l=1}^{L} \alpha_{l n} \sqrt{P_{l n}} h_{l n m} w_{l n}\right|^{2}}, \quad \forall m
\end{aligned}
$$

where the vectors $\alpha$ and $\mathrm{w}$ respectively represent the set of coefficients for the power coefficients $\alpha_{l m}$ and the beam steering coefficients $w_{l m}$.

Transmission strategy is based on maximizing the minimum SINR over all users. This scenario can be formulated as an optimization problem with port power coefficients and beamsteering coefficients as the optimization variables

$$
\begin{array}{ll}
\max _{\boldsymbol{\alpha}, \mathbf{w}} & \min _{m} \operatorname{SINR}_{m}(\boldsymbol{\alpha}, \mathbf{w}) \\
\text { s.t. } & \boldsymbol{\alpha} \in\{0,1\}^{L M}
\end{array}
$$

for binary power management, and

$$
\begin{array}{ll}
\max _{\boldsymbol{\alpha}, \mathbf{w}} & \min _{m} \operatorname{SINR}_{m}(\boldsymbol{\alpha}, \mathbf{w}) \\
\text { s.t. } & \boldsymbol{\alpha} \in[0,1]^{L M}
\end{array}
$$

for continuous power management. Both problems are nonconvex and it can be difficult to solve on the search space $(\boldsymbol{\alpha}, \mathbf{w})$ as the number of cells increase.

Since a port utilizes a single antenna, its beam steering coefficient is composed of a complex-valued scalar. In order to simplify the above optimization problem, it is assumed that beam steering coefficients are chosen to match the phases of the channel between the ports and their intended UEs

$$
w_{l m} \triangleq e^{-j \angle h_{l m m}} \quad \forall l, m .
$$

Although under this assumption the solution becomes suboptimal, it has been shown that this approach maximizes the SINR when the interference power is assumed to be fixed [4]. 
In the new formulation, the problem is reduced to port selection for BPM and adjusting the power levels of the ports for CPM, i.e.,

$$
\begin{aligned}
\max _{\boldsymbol{\alpha}} & \min _{m} \operatorname{SINR}_{m}(\boldsymbol{\alpha}), \\
\text { s.t. } & \boldsymbol{\alpha} \in\{0,1\}^{L M}, \quad \text { for BPM } \\
\max _{\boldsymbol{\alpha}} & \min _{m} \operatorname{SINR}_{m}(\boldsymbol{\alpha}), \\
\text { s.t. } & \boldsymbol{\alpha} \in[0,1]^{L M}, \quad \text { for CPM. }
\end{aligned}
$$

Clearly, problem (4a) is a non-linear binary-integer programming problem and problem (4b) can be shown to have a multi-modal cost surface. By using conventional optimization algorithms, i.e., convex optimization, the first problem can only be solved by using integer-relaxation which will possibly yield a suboptimal solution. Furthermore, since the second problem is multi-modal, a conventional solver may get stuck at a local optimum solution. In order to overcome these problems we propose to use Particle Swarm Optimization (PSO) as a solver.

\section{PARTIClE SWARM Optimization}

PSO [8] is a stochastic evolutionary optimization algorithm which mimics the behavior of swarms (e.g., bees). Some of the prominent features of PSO are ease of implementation, low computational complexity, and ability to find the global solution in a multimodel problem. These features makes PSO a good candidate to solve the problems at hand.

The PSO algorithm is initialized with a population of random candidate solutions, $\mathbf{x}_{i}$, namely particles. PSO finds optimal regions of complex search spaces through the interaction of individuals in the population of these particles. Each particle is initially assigned a randomized velocity, $\mathbf{v}_{i}$, and is iteratively moved through the problem space. It is attracted towards the location of the best fitness achieved so far by the particle itself, $\mathbf{p}_{i}$, and by the location of the best fitness achieved so far across the whole population, $g$ [9]. The parameters, $c_{l}, c_{g}$ - called acceleration coefficients - control the behavior and efficacy of the algorithm and are chosen heuristically. The variables $r_{l}$ and $r_{g}$ are random positive numbers, drawn from a uniform distribution. The pseudocode of the algorithm is given below:

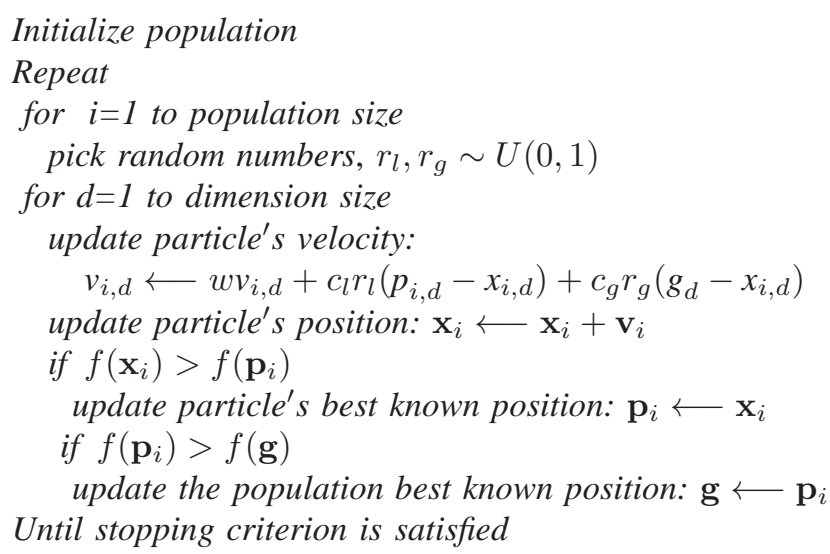

In our optimization problems, we use binary PSO [10] for binary power management whereas traditional PSO is used for continuous power management. We use 60 swarms and 1000 swarms for population size in two-cell cluster and seven-cell cluster, respectively. We take the inertia coefficient as $w=1$, and acceleration coefficients as $c_{l}=0.85$ and $c_{g}=2$.

\section{COMPlEXity ANALYSis}

In this Section, complexity of the proposed methods will be analyzed. In the case of binary power management, exhaustive search is done by searching all possible port states and has a computational complexity $O\left(2^{L M}\right)$, hence for large $L$ and $M$ this method is inefficient. By considering the algorithm in Section III, particle swarm optimization has a computational complexity in the order of $O(S L M N)$ where $S, L, M$ and $N$ denote the population size, number of ports in each cell, number of cells and number of iterations done until stopping criterion is satisfied, respectively.

For the stopping criterion, in CPM, iterations continue until all SINR values are contained in a neighbourhood of $\mp 0.05 \mathrm{~dB}$, whereas in BPM, iterations continue until no change in the particle positions is observed for 20 consecutive iterations. However, it should be noted that the complexity of BPM is higher than CPM since the number of iterations and computational complexity of the update equation in BPM is higher. When there is one swarm, update equation has 6 multiplications in CPM whereas there are 7 multiplications and 1 exponentiation (comes from the sigmoud function in probabilistic update equation [10]) in BPM, which increases computational complexity.

For the case of two-cell network, it is found that the average number of iterations for BPM and CPM are 53 and 21, respectively, whereas for the seven-cell cluster, the average number of iterations for BPM and CPM are 97 and 27, respectively.

\section{Performance Evaluation}

We analyze the performance of the proposed method for a network of $M$ hexagonal cells and $L=7$ ports per cell through Monte Carlo simulations. One of the ports is located at the center of the cell, and others are located uniformly at a distance of $2 / 3$ of the circumradius $\left(r_{c}\right)$ from the center, in order to increase the coverage of the cell. Ports can either be set off, or they can transmit at a fixed power level of $P_{\max }$ for BPM, or they can transmit at an adjustable power level in the interval $\left[0, P_{\max }\right]$ for the CPM.

For the simulations we consider a single RB throughout the network. As it is stated above, at most one UE can use this RB in a cell, therefore $M$ UEs are considered network-wide, and these UEs are located randomly in each cell for each channel realization.

For the port-to-UE link, a Rayleigh fading channel with log-normal shadowing and path loss components as in [11] are considered. The complex channel gains are $h_{\operatorname{lnm}}=$ $\sqrt{\rho\left(d_{l n m}\right) s_{\operatorname{lnm}}} h_{l n m}^{\prime}$, where $\rho(\cdot)$ is the path loss function given below, $d_{l n m}$ is the distance between the $l$-th port of the $n$-th 
cell and the user in the $m$-th cell, $s_{l m n}$ represents log-normal shadowing with $0 \mathrm{~dB}$ mean and $8 \mathrm{~dB}$ standard deviation, and $h_{l n m}^{\prime}$ denotes the fading effect and has a complex Gaussian distribution with zero mean and unit variance. For the suburban scenario described in [11], the distance between base stations is $1299 \mathrm{~m}$, and the noise power is $-114 \mathrm{dBm}$. The path loss function considered here is

$$
\rho\left(d_{\operatorname{lnm}}\right)=10^{-\left(1.866+4.032 \log _{10}\left(d_{\ln m}\right)\right)}
$$

where carrier frequency is taken as $2 \mathrm{GHz}$, antenna ports are at a height of $15 \mathrm{~m}$, and each UE is assumed to have $1.5 \mathrm{~m}$ elevation.

In order to assess the optimality of the proposed algorithm based on PSO, we first consider a two-cell sub-urban macrocell scenario. One UE is randomly dropped in each cell. Each cell has $L=7$ ports. Minimum SINR in the cluster is maximized by the PSO based algorithm for both binary and continuous power management scenarios given respectively by the problems (4a) and (4b). For comparison purposes, exhaustive search is also performed for binary power management, which searches the best result out of $2^{14}$ port state vectors. The results depicted in Fig.s 2 and 3 are averaged over 300 realizations.

Fig. 2 compares the resulting SINR levels with respect to the maximum port power limit $P_{\max }$ for both the BPM and CPM schemes. It can be seen that for lower $P_{\max }$ values, both the BPM and CPM results yield almost the same performance. This is the noise-limited region in which one does not gain much with power management due to negligible interference from other cells. As power limit increases, e.g., higher than $30 \mathrm{dBm}, \mathrm{CPM}$ becomes to outperform BPM, and after approximately $P_{\max }=55 \mathrm{dBm}$ a noise floor effect is observed limiting the performance of BPM. The region $P_{\max } \gtrsim 30 \mathrm{dBm}$ can be considered as the interference limited region, in which interference from other users dominate the AWGN in the channel. In this region, rather than just switching the ports on and off (BPM), adjusting the transmission power of them (CPM) appears to perform better. As it can be seen from Fig. 2 at $P_{\max }=40 \mathrm{dBm}$, the UE with minimum SINR gains $5 \mathrm{~dB}$ by CPM.

From Fig. 2, it should be noted that for the BPM scheme, PSO performs almost the same as exhaustive search, verifying the optimality of the PSO algorithm. This is an important aspect of PSO since PSO can optimize complex problems with lower computation time. Obtaining to the optimum solution of the CPM scenario is difficult by evaluating with exhaustive search, and it is not possible to find the global optimum by using a conventional optimization algorithm. Therefore we cannot compare the CPM result with PSO to that of an global optimizer.

In Fig. 3, the average transmit power per port vs. the maximum port power limit $P_{\max }$ is compared for both the BPM and CPM schemes. According to the figure, both schemes perform similar, and they both require similar total transmit power per port for the same $P_{\max }$ values. In other words, for the

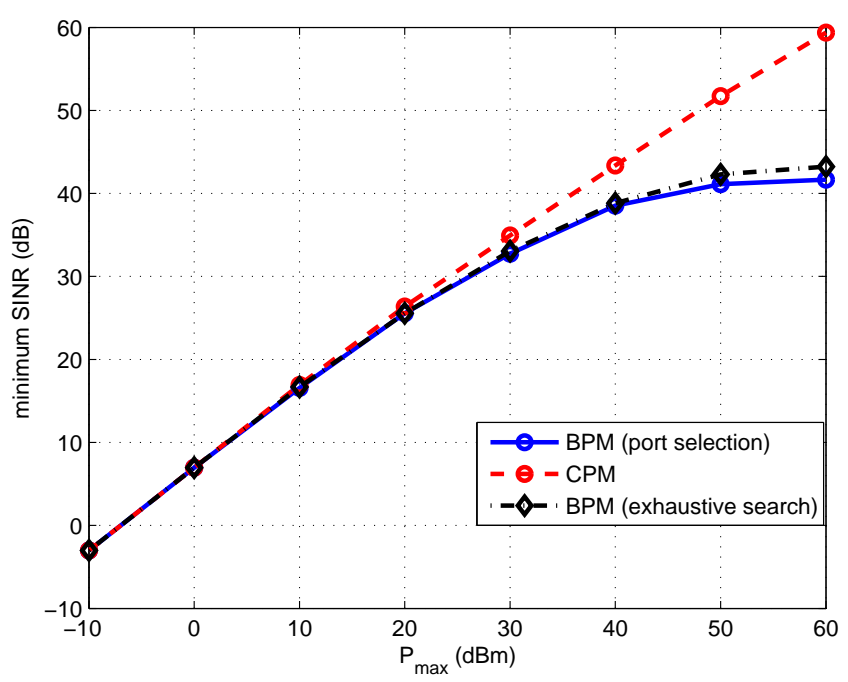

Fig. 2: Largest minimum SINR achieved by binary power management and continuous power management for a two-cell cluster. Solid, dashed and dotdashed line represents BPM (port selection), CPM and BPM (exhaustive search), respectively.

same total transmit power per port levels, CPM yields higher minimum SINR values as compared to BPM. This result demonstrates the advantage of using CPM in the interference limited region. For example, for $P_{\max }=60 \mathrm{dBm}$, although CPM requires the same average transmit power per port, it results in more than $15 \mathrm{~dB}$ gain in SINR as compared to BPM.

Similar to Fig. 2, Fig. 3 also demonstrates that PSO performs almost the same as exhaustive search for the BPM scheme, verifying the optimality of solution of the PSO algorithm.

When we increase the scale of the problem, the advantage of CPM over BPM becomes more apparent. In the next set of simulations we consider a sub-urban macro-cell scenario with 7 cells and 7 ports per cell with the same settings as in the above study. Fig. 4 depicts that the curve for CPM and BPM are separated for almost all $P_{\max }$ values. Furthermore, Fig. 5 shows that for all $P_{\max }$ values even though CPM and BPM require the same $P_{\max }$ level to obtain the same total transmit power per port, CPM outperforms BPM in terms of the minimum SINR in the network. Considering a range for $P_{\max }$ from $-10 \mathrm{dBm}$ to $30 \mathrm{dBm}$ as a typical operation condition, one can deduce that for a typical cellular communications scenario with moderate number of cells, CPM is more advantageous than BPM.

\section{CONCLUSION}

In this paper, a coordinated multi-cell distributed antenna system is considered. In order to increase the coverage and throughput of the network, instead of employing a single base station in a cell, a number of ports are distributed throughout the cell which transmit the same signal.

Two transmission schemes are investigated; either the ports are switched on and off (Binary Power Management, BPM) 


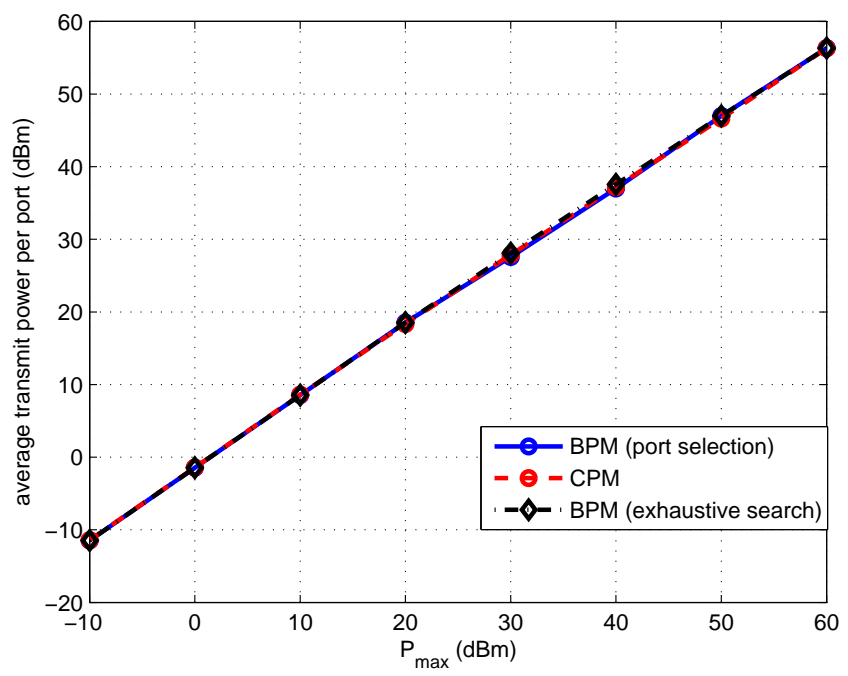

Fig. 3: Average transmit power per port achieved by binary power management and continuous power management for a two-cell cluster. Solid, dashed and dotdashed line represents BPM (port selection), CPM and BPM (exhaustive search), respectively.

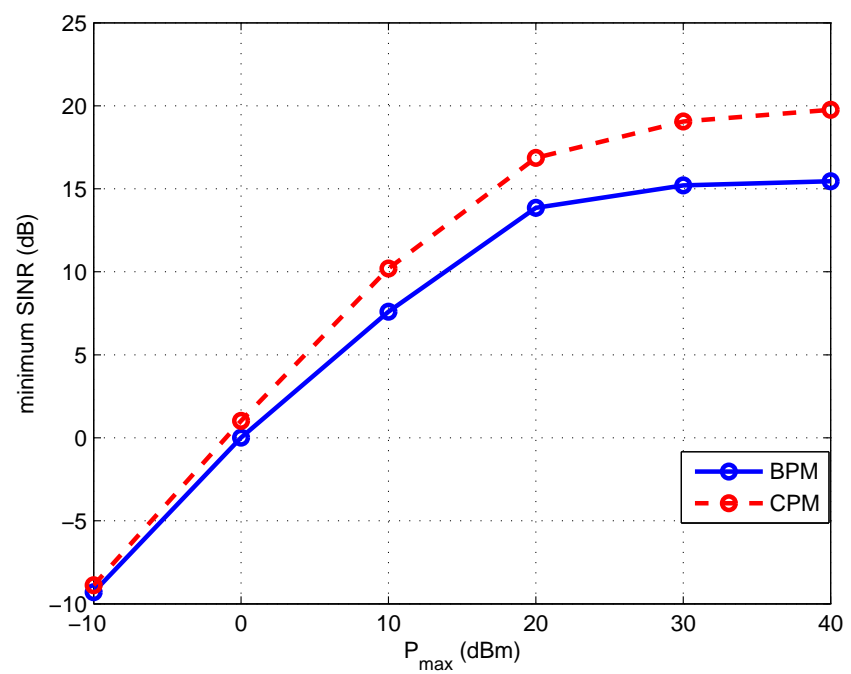

Fig. 4: Largest minimum SINR achieved by binary power management and continuous power management for a seven-cell cluster. Solid and dashed line represents BPM and CPM, respectively.

with a maximum transmit power limit per port or they are allowed to transmit with variable power (Continuous Power Management, CPM). It has been demonstrated that for a small sized network, CPM outperforms BPM especially in the interference-limited region, where interference to a user originates from the ports of other cells. It is also shown that, for a more complex network with a higher number of cells, CPM performs better than BPM under practically meaningful conditions. Proper power management introduces an important gain to system performance, which can be considered very

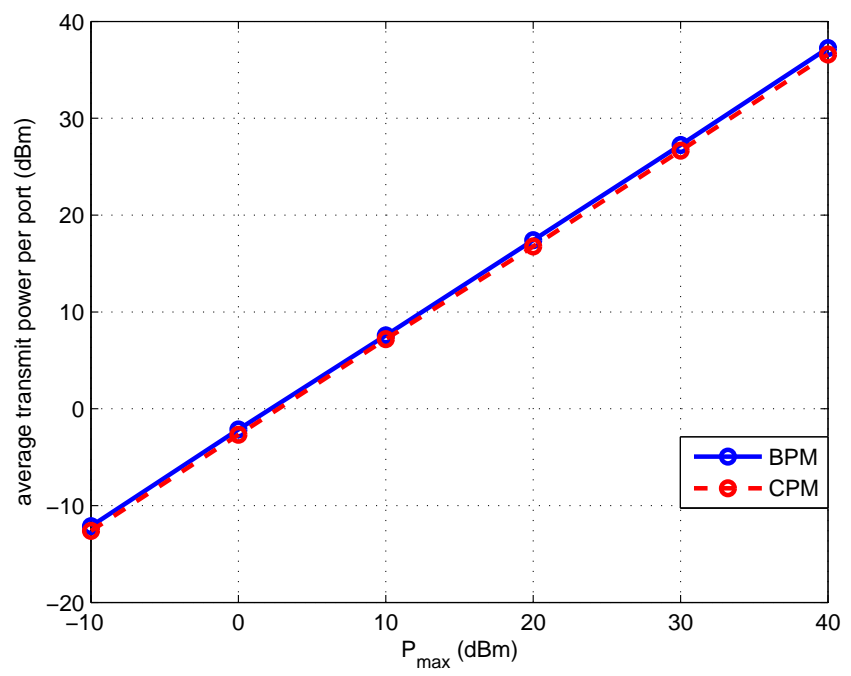

Fig. 5: Average transmit power per port achieved by binary power management and continuous power management for a seven-cell cluster. Solid and dashed line represents BPM and CPM, respectively.

promising for next generation networks.

It is observed that the implementation complexity of CPM when using PSO is lower than that of BPM, making CPM more advantageous than BPM in both performance and also complexity aspects.

\section{REFERENCES}

[1] S. Venkatesan A. Lozano, and R. Valenzuela, "Network MIMO: overcoming intercell interference in indoor wireless systems," Proc. 41st Asilomar Conf. Signals, Systems and Computers, pp. 83-87, Nov. 2007.

[2] R. Bendlin, V. Chandrasekhar, R. Chen, A. Ekpenyong, and E. Onggosanusi, "From homogeneous to heterogeneous networks: a 3GPP long term evolution rel. 8/9 case study," Proc. 45th Annu. Conf. Information Sciences and Systems (CISS), pp. 1-5, Mar. 2011.

[3] T. Ahmad, R. H. Gohary, H. Yanikomeroglu, S. Al-Ahmadi, and G. Boudreau, "Coordinated port selection and beam steering optimization in a multi-cell distributed antenna system using semidefinite relaxation," IEEE Trans. Wireless Commun., vol. 11, no. 5, pp. 18611871, May 2012.

[4] W. Choi and J. Andrews, "Downlink performance and capacity of distributed antenna systems in a multicell environment," IEEE Trans. Wireless Commun., vol. 6, no. 1, pp. 69-73, Jan. 2007.

[5] L. Vanderberghe and S. Boyd, "Semidefinite programming," SIAM Rev., vol. 38, pp. 49-95, Mar. 1996.

[6] Z. Q. Luo, W. K. Ma, A. C. So, Y. Ye, and S. Zhang, "Semidefinite relaxation of quadratic optimization problems," IEEE Signal Process. Mag., vol. 27, pp. 20-34, May 2010.

[7] M. Grant and S. Boyd, "CVX: Matlab software for disciplined convex programming, version 1.21," Jan. 2011. Available: http://cvxr.com/cvx.

[8] J. Kennedy and R. Eberhart, "Particle swarm optimization," Proc. IEEE Int. Conf. Neural Networks, Piscataway, NJ, USA, vol. 4, pp. 1942-1948, 1995.

[9] I. C. Trelea, "The particle swarm optimization algorithm: convergence analysis and parameter selection," Information Processing Letters 85, pp. 317-325, 2003.

[10] F. V. D. Bergh, "An analysis of particle swarm optimizers," Ph.D. dissertation, University of Pretoria etd, Pretoria, South Africa, 2001.

[11] International Telecommunication Union (ITU), "Guidelines for evaluation of radio interface technologies for IMT-Advanced," TR M.2135-1, ITU-R, Dec. 2009. Available: http://www.itu.int/pub/R-REP-M. 2135-12009. 